\title{
Control interpretations of products in the Hopf algebra
}

\author{
Matthias Kawski
}

\begin{abstract}
Families of differential operators, like those defining affine, generally nonlinear, control systems are known to have natural Hopf algebra structures. These provide deeper insight into relationships and properties of such objects as the Chen-Fliess series and state space realizations of systems defined by input-output operators. A starting point for this work are representations of control objects by formal powerseries in, generally noncommuting, indeterminates. Such series are elements of a free associative algebra which contains several rich substructure that are equipped with various products. This article summarizes correspondences between objects from control theory and algebraic combinatorics, with special focus on elements of the two Hopf algebra structures on this algebra of formal power series, including the antipode and two convolution products. Newly found relationships are discussed, and open questions are posed. Recent work has demonstrated that these abstract combinatorial algebra structures help obtain effective solution formulas for various control problems including questions about controllability and algorithms for path planning. They also find applications in the field of geometric numerical integration algorithms.
\end{abstract}

\section{INTRODUCTION}

Transform techniques and asymptotic series expansions have a long history in the field of dynamical systems. In the realm of linear systems, Laplace transforms of both signals and systems not only provide an algebraic setting suitable for effective manipulation and computations, but also a powerful conceptual language. For nonlinear systems, different commonly used tools are asymptotic expansions that trace their roots to Volterra series introduced in the late 1800s. In the setting of nonlinear control systems, that are affine in the control, the Chen Fliess series takes a particularly prominent role. Its roots in combinatorial algebra trace back to the work of Chen [1] who associated formal power series to curves in Euclidean space for the purpose of studying their geometric invariants. Fliess [2], [3], [4] recognized the utility of this series for control theory, especially for relating input-outputoperator approaches and state-space realizations, with much closely related work by e.g. Crouch [5], Jakubczyk [6], [7], [8], and many others. Convergence issues have been analyzed widely, compare e.g. Sussmann [9], Wang and Sontag [10], Gray and Wang [11]. The work presented is concerned with combinatorial and algebraic aspects.

This work was partially supported by the National Science Foundation through DMS 05-09030.

M. Kawski is with the School of Mathematical and Statistical Sciences, Arizona State University, Tempe, AZ 85287. kawski@asu. edu
The central role of the shuffle product for establishing that the Chen series is an exponential Lie series was recognized by Ree [12]. The factorization of the series into an infinite directed product of exponentials by Sussmann [13] introduced a different multiplicative structure, which since has been studied in more abstract contexts under the name of a Zinbiel algebra [14], [15]. Implicitly this product already was already utilized by Schützenberger [16]. More recently, EbrahimiFard, Manchus, and Patras, [17], [18] demonstrated that this product is only a part of a more fundamental underlying dendriform algebra.

Grossman and Larson [19] used Hopf algebras for the realization of input-output maps, see also [20], [21], [22] for related work on Hopf algebraic structures of families of differential operators. A Hopf algebraic approach is taken in the study of free Lie algebras by Reutenauer [23] which comments on many close connections to control theory. Yet another product appears under the name of composition product in the work by Gray and Li [24], and especially in [25], which considers formal Laplace-Borel transforms and Fliess operators. Closely related work by Gray and Wang [26] also analyzes noncausal Fliess operators.

Apart from realization theory, Fliess series are also integral tools in the study of nonlinear controllability and optimality, see e.g. [27], [28], [29] and in path planning algorithms, e.g. [30], [31]. Important applications outside control include geometric numerical integration of ordinary differential equations with algebraic constraints, see e.g. [32].

The article emphasizes throughout the chronological point of view introduced to control by Agrachev and Gamkrelidze [33], [34], which concentrates on the action of flows on the algebra of smooth functions on a manifold (rather than on points on the manifold), see also [35]. The subsequent sections exhibit many different points of view of the Fliess series, review central objects in Hopf algebras, and then demonstrate the correspondences between combinatorial objects and objects in control theory, with special emphasis on various product and (semi-)group structures. The focus of this article is on algebraic relationships, referring to above mentioned works for convergence and continuity aspects of the maps.

\section{MANy FliEsS SERIES}

Consider an affine control system on a manifold $M^{n}$ in state space form 


$$
\begin{aligned}
\dot{x} & =\sum_{i=1}^{n} u^{i} f_{i}(x) \\
y & =\varphi(x)
\end{aligned}
$$

with output function $\varphi$, analytic vector fields $f_{i}$ on $M$, and measurable controls $u$ taking values in compact subset of $\mathbb{R}^{m}$, and which is initialized at $x_{0} \in M$. Write $\mathcal{U}$ for the set of admissible controls. [In many settings of interest one adds an uncontrolled drift vector field $f_{0}$ with corresponding fixed coefficient $u^{0} \equiv 1$.] For any multi-index $I=\left(i_{1}, \ldots, i_{s}\right) \in\{1, \ldots m\}^{s}$ with $s \geq 0$, introduce the $s$-order partial differential operator

$$
f_{I}=f_{i_{1}} f_{i_{2}} \ldots f_{i_{s}}
$$

and for $u \in \mathcal{U}$ and $I$ as above introduce the iterated integral

$$
\Upsilon^{I}(t, u)=\int_{0}^{t} \int_{0}^{t_{1}} \cdots \int_{0}^{t_{p-1}} u^{i_{s}}\left(t_{s}\right) \cdots u^{i_{1}}\left(t_{1}\right) d t_{1} \ldots d t_{s} .
$$

Then for every compact set $K \subseteq M$ of initial conditions, there exists a $T>0$ such that for all $t \in[0, T]$ the series

$$
(\operatorname{Sep}(t, u) \varphi)\left(x_{0}\right)=\sum_{I} \Upsilon^{I}(t, u)\left(f_{I} \varphi\right)\left(x_{0}\right)
$$

converges uniformly to $\varphi$, compare e.g. [9].

There are many ways to read this series: As written above, it is simply a Taylor-like expansion of the output $y(t)$ of a control system for given system data $f_{1}, \ldots f_{m}, \varphi, x_{0}$ and control $u$. In the analysis of optimal control problems one frequently also fixes the time $t=T$ and considers the resulting endpoint map with $u \in \mathcal{U}$ being the variable of interest. Considering $x_{0} \in K \subseteq M=\mathbb{R}^{n}$ and $t \in[0, T]$ variable, the identity (5) with $\varphi(x)=x$, the identity-map on the state-space $M$, is interpreted as an expansion of the flow corresponding to a fixed control $u$. Alternatively, from the chronological point of view, that expression defines a oneparameter family of automorphisms on the algebra $C^{\infty}(M)$ of smooth functions on $M$.

Further abstracting, on one side one may consider the control $u$ fixed, but the system data $f_{1}, \ldots, f_{m}$ and $\varphi$ to be undetermined. If there is no given output function $\varphi$, this series becomes an (unevaluated) series of partial differential operators of arbitrarily high order. In the literature one commonly finds analogous expressions with (noncommuting) indeterminates $X_{i}$ taking the place of the symbols $f_{i}$ in (1). In particular, this associates to every control $u$ a formal power series in noncommuting indeterminates $X_{1}, \ldots X_{m}$. In algebraic combinatorics, it is common to simplify the notation by working only with the index $i$ of the indeterminate $X_{i}$, and call $i$ a letter. Generally the letters are assumed to be taken from a set $Z$ rather than required to be elements of $\{1, \ldots m\}$.
Conversely, one may consider a fixed system with given $f_{1}, \ldots, f_{m}$ and $\varphi$ and consider the control $u \in \mathcal{U}$ the main variable of interest, and then obtains an input-output relationship that maps the input signal $u(\cdot)$ to the output signal $y(\cdot)$. In the most simple form of the state-space realization problem, this operator is considered to be the given data, one asks under what conditions one can find $M, f_{1}, \ldots f_{m}, \varphi$ which represent the operator in the above form. More formally, the series associated to the system is a generally infinite linear combination of iterated integral operators $\Upsilon^{I}$ (with (time $t$ fixed or variable). Again, for many calculations the nature of $\Upsilon^{I}$ as an iterated integral operator is immaterial, and one may simply work with the word $I=i_{1} i_{2} \ldots i_{s}$, albeit taking into consideration the natural multiplicative structures satisfied by these iterated integral functionals. (As elaborated below, the characteristic product structure defines a nonassociative Zinbiel algebra.) In particular, this point of view associates to every system $f_{1}, \ldots f_{m}$ (without output function) a formal power series.

Further abstracting, by considering all data as variables, and writing simply words $I$ for both the noncommutative polynomials $X_{I}$ and iterated integral functionals $\Upsilon^{I}$, the series reduces to the formal expression

$$
\sum_{I} I \otimes I
$$

where the sum is taken over all words (or multi-indices) $I \in Z^{*}=\bigcup_{s=0}^{\infty} Z^{s}$. Considering the set of all words as a basis for the free associative algebra $A(Z)$ on the set $Z$ of letters (over the field of the real numbers), one may interpret this sum simply as a representation of the identity map on $A(Z)$. This uses the natural identification of the space $\operatorname{Hom}(A(Z), A(Z))$ of linear endomorphisms of $A(Z)$ with $A(Z) \otimes \hat{A}(Z)$, where $\hat{A}(Z)$ may here be understood as the associative algebra of noncommutative formal powerseries and $A(Z)$ is the set of noncommutative polynomials (see the next section for details). This establishes the Fliess series as in (5) as the image under various evaluation homomorphisms of a natural object, the identity on a free algebra. The remainder of this article is devoted to studying the nature of the various algebra structures and homomorphisms, and relating these to operations on systems and controls, respectively. In particular, both systems and controls are represented by series, and there are multiple ways in which pairs consisting of a system and a control, of two systems, or of two controls can be meaningfully combined. Moreover, while every system such as (1) and every admissible control in $\mathcal{U}$ corresponds to a formal power series, the converse is not true. This indicates a need to characterize which formal power series correspond to controls or systems of the usual form (this is a major theme of the literature on realization theory), but also suggests the possibility of 
defining generalized systems and generalized controls which still share key algebraic properties, but which may not be representable in the above form. The technical details of this endeavor, including judicious choices of suitable topologies, notions of convergence and of approximations by classical controls or classical systems are far beyond the scope of the current article.

\section{HOPF ALGEBRA AND CONVOLUTION PRODUCTS}

This section reviews basic elements from combinatorial Hopf algebras that are used in the sequel. For further details the reader is referred to [23]. From now on, rather than using natural numbers $i \in\{1, \ldots m\}$ as indices for vector fields, and coordinates of the controls, we follow the combinatorics literature, and use letters taken from a set $Z$, called an alphabet (here taken to be finite). Correspondingly, in this notation, multi-indices $\left(a_{1}, \ldots, a_{s}\right)$ are collapsed into strings $a_{1} a_{2} \ldots a_{s}$ and are called words.

The linear space $A(Z)$ of all noncommutative polynomials (words) in the letters of the set $Z$, with coefficients in the field $\mathbb{R}$, has a natural associative product structure as the bilinear extension of concatenation of words conc: $\left(a_{1}, \ldots a_{r}, b_{1} \ldots b_{s}\right) \mapsto a_{1}, \ldots a_{r} b_{1} \ldots b_{s}$. The space $A(Z)$ also has a natural inner product $\langle\cdot, \cdot\rangle$ which is such that the set $Z^{*}$ of all words is an orthonormal basis. Write $e$ for the empty word, which satisfies $w e=w=e w$ for every word $w \in Z^{*}$.

The coproduct $\Delta: A(Z) \mapsto A(Z) \otimes A(Z)$ is the concatenation-algebra homomorphism defined on letters $a \in Z$ by

$$
\Delta(a)=e \otimes a+a \otimes e .
$$

For example, for letters $a, b \in Z$, one has

$$
\Delta(a b)=e \otimes a b+a \otimes b+b \otimes a+a b \otimes e .
$$

The transpose of this coproduct is the shuffle product ш : $A(Z) \times A(Z) \mapsto A(Z)$ defined for words $u, v, w \in A(Z)$ by

$$
\langle u ш v, w\rangle=\langle u \otimes v, \Delta w\rangle .
$$

Recursively, it is characterized for letters $a, b \in A$ and words $w, z \in A(Z)$ by $(w a) ш(z b)=((w a) ш z) b+(w ш(z b)) a$. One readily verifies that the shuffle product equips $A(Z)$ with a commutative associative algebra structure.

Using this structure, one may define a second coproduct $\Delta^{\prime}$ on $A(Z)$ as the shuffle-algebra homomorphism which satisfies on letters the same defining relation

$$
\Delta^{\prime}(a)=e \otimes a+a \otimes e .
$$

For example, for letters $a, b \in Z$, one has

$$
\Delta^{\prime}(a b)=e \otimes a b+a \otimes b+a b \otimes e .
$$

Finally define a linear map $\alpha: A \mapsto A$, that maps any word $w=a_{1} a_{2} \ldots a_{k} \in Z^{k}$ to its signed reversal $w=$ $(-)^{k} a_{k} \ldots a_{2} a_{1} \in Z^{k}$. It is well known that the linear space $A(Z)$ is endowed with two Hopf algebra structures whose triples of product, coproduct and antipode are given by (conc, $\Delta, \alpha)$ and $\left(\omega, \Delta^{\prime}, \alpha\right)$, respectively.

One naturally topologizes $A(Z)$ by defining a uniform structure in terms of a basis of entourages of the identity which is given by all possible sets pairs of polynomials that which have identical coefficients on any given finite set of words, for details see [15], [23]. The corresponding completion of $A(Z)$ is denoted by $\hat{A}(Z)$. Since the products defined above are uniformly continuous, they extend continuously to $\hat{A}(Z) \times \hat{A}(Z)$.

The space $\operatorname{Hom}(A(Z), A(Z))$ of linear endomorphisms of $A(Z)$ has the standard associative algebra structure defined by compositions of endomorphisms. But it also has two other associative algebra structures with convolution products $\star, \star^{\prime}$ defined on linear endomorphisms $\Phi, \Psi: A(Z) \mapsto A(Z)$ by

$$
\begin{aligned}
\Phi \star \Psi & =\operatorname{conc} \circ(\Phi \otimes \Psi) \circ \Delta, \text { and } \\
\Phi \star^{\prime} \Psi & =\operatorname{shu} \circ(\Phi \otimes \Psi) \circ \Delta^{\prime},
\end{aligned}
$$

i. e., for any word $w \in A(Z)$

$$
\begin{aligned}
& (\Phi \star \Psi)(w)=\sum_{u, v \in Z^{+}}\langle w, u ш v\rangle \Phi(u) \Psi(v) \text { and } \\
& \left(\Phi \star^{\prime} \Psi\right)(w)=\sum_{u, v \in Z^{+}}\langle w, u v\rangle \Phi(u) ш \Psi(v) \\
& =\sum_{\substack{u v=w \\
u, v \in Z^{+}}} \Phi(u) ш \Psi(v) .
\end{aligned}
$$

When equipping the complete tensor product $A(Z) \bar{\otimes} A(Z)$ with the shuffle product on the left and the concatenation product on the right, then the canonical imbeddings $\operatorname{Hom}(A(Z), A(Z)) \mapsto A(Z) \bar{\otimes} A(Z)$ defined by

$$
\begin{aligned}
& \Phi \mapsto \sum_{w \in Z^{*}} w \otimes \Phi(w) \quad \text { and } \\
& \Phi \mapsto \sum_{w \in Z^{*}} \Phi(w) \otimes w
\end{aligned}
$$

algebra homomorphisms for the convolution products $\star$ and $\star^{\prime}$, respectively, compare proposition 1.10 in [23]. The unit in the algebra $A(Z) \bar{\otimes} A(Z)$ is the map which maps every polynomial in $A(Z)$ to its constant term, and the inverse of the identity on $A(Z)$ in this algebra is the antipode $\alpha$.

Finally write $I$ for the map which subtracts from every polynomial its constant term, i.e., $I(w)=w-\langle 1, w\rangle$.

\section{SERIES OF CONTROLS AND THEIR PRODUCTS}

For the sake of clarity of exposition let us specialize to the case of controls $u$ taking values in the cube $[-1,1]^{m}$ and redefine $\mathcal{U}$ as the space of all absolutely continuous functions 
$U:[0, T] \mapsto[0,1]$, considered as the primitives $\int_{0}^{t} u^{a}(s) d s$ of the coordinate functions of the controls in the introductory second section. Start with the identity map on $A(Z)$ written in the form $\sum_{w \in Z^{*}} w \otimes w$ as the most abstracted form of the Chen-Fliess series.

Technically, it might be even more appropriate to start with the inverse (with respect to the convolution product) $\sum_{w \in Z^{*}} w \otimes \alpha(w)$ of the identity on $A(Z)$, which corresponds even more naturally to the evaluation of the words $w$ on partial differential operators $f_{w}$. But here we will employ a suitable anti-automorphism, taking the place of $\alpha$ above) at a different place.

As a first step, map this expression to a formal power series whose coefficients are iterated integral functionals, that is elements of a special form in the larger space of all mappings from the space $\mathcal{U}^{m}$ to $\mathcal{U}$.

$$
\mathrm{CF}=(\Upsilon \otimes \mathrm{Id})\left(\sum_{w \in Z^{*}} w \otimes w\right)=\sum_{w \in Z^{*}} \Upsilon^{w} \otimes w
$$

This is the Chen-Fliess series in the form considered in much of the literature on controllability, optimality, path-planning and the like.

The fact that the map $\Upsilon$ is a associative algebra homomorphism from $A(Z)$ equipped with the shuffle product to the algebra of iterated integral functionals with the product of pointwise multiplication is effectively known as Ree's theorem [12], and it guarantees that this series is an exponential Lie series with iterated integral coefficients.

However, more than just a cosmetic flaw of this product is that the pointwise product of two iterated integral functionals is not readily recognizable as a linear combination of iterated integral functionals of the form as in (4).

Indeed, the natural product in control theory for absolutely continuous functions $U, V:[0, T] \mapsto \mathbb{R}$ is

$$
(U * V)(t)=\int_{0}^{t} U(s) \cdot V^{\prime}(s) d s .
$$

This product has an abstract combinatorial counterpart. Indeed, the shuffle product is the symmetrization (and extension) of the Zinbiel product $*: A^{+}(Z) \times A(Z) \mapsto A(Z)$ defined as the bilinear extension to $A(Z)^{+} \times A(Z)$ of the product defined for nonempty words $w, z \in Z^{*} \backslash\{e\}$, and any letter $a \in Z$ by $e * w=w, w * a=w a$, and

$$
w *(z a)=(w * z) a+(z * w) a
$$

Note that $e * e$ remains undefined. One readily verifies that both products (17) and (18) satisfy the right Zinbiel identity

$$
r *(s * t)=(r * s) * t+(s * r) * t .
$$

It is easily verified that the map $\Upsilon$ is a Zinbiel algebra homomorphism [15], [36].
For the sake of facilitating the exposition, let us now fix a control $u=U^{\prime}$ where $U=\left(U^{1}, \ldots U^{m}\right) \in \mathcal{U}^{m}$. Consider the Fliess series $C F(u)$ of that control as a curve in the algebra $A(Z)$

$$
\mathrm{CF}(u)=\sum_{w \in Z^{*}} \Upsilon^{w}(u) \cdot w:[0, T] \mapsto A(Z) .
$$

By the Friederich's criterion this curve of exponential Lie series (with coefficients that are absolutely continuous scalar functions, i.e. elements in $\mathcal{U}$ ) is a group-like element in $A(Z)$, i.e. it satisfies

$$
\Delta(\mathrm{CF}(u))=\mathrm{CF}(u) \otimes \mathrm{CF}(u) .
$$

The subset of all such group-like elements forms a formal group. In particular, it is closed under the multiplication. It is known [9] that this multiplication of the series of controls correspond to the concatenation of controls, i.e. if $u:\left[0, T_{1}\right] \mapsto[-1,1]^{m}, v:\left[0, T_{2}\right] \mapsto[-1,1]^{m}$, and $(u \sharp v):\left[0, T_{1}\right] \mapsto[-1,1]^{m}$ is defined by $(u \sharp v)(t)=u(t)$ if $t \in\left[0, T_{2}\right]$ and $(u \sharp v)(t)=v\left(t-T_{1}\right)$ if $t \in\left(T_{1}, T_{1}+T_{2}\right]$, then

$$
\mathrm{CF}(u \sharp v)=\mathrm{CF}(v) \cdot \mathrm{CF}(u) .
$$

In other words $\mathrm{CF}$ is a semi-group homomorphism from the space of controls under the product of concatenation to the formal group of exponential Lie series. The product structure of the latter is an important building block for control applications such as path planning algorithms.

Indeed, suppose $\mathcal{H}$ is a basis for the free Lie algebra $L(Z)$ over the set $Z$. Such bases are easily recursively constructed, compare e.g. [15] for a survey and numerous examples. Its elements $\ell \in \mathcal{H}$, called Lie polynomials, are primitive in the sense that $\Delta(\ell)=\ell \otimes 1+1 \otimes \ell$. Thus it is a-priori clear that there exists maps $\xi, \zeta$ that maps the set $\mathcal{H}$ (actually, in the case of Hall bases one may use a simpler underlying set of Hall word, compare [36]) into the space of iterated integral functionals such that

$$
\mathrm{CF}=\exp \left(\sum_{\ell \in \mathcal{H}} \zeta_{\ell} \otimes \ell\right)=\overleftarrow{\prod_{\ell \in \mathcal{H}}} \exp \left(\xi_{\ell} \otimes \ell\right) .
$$

Explicit expressions for the iterated integral functionals $\xi_{\ell}$ in the setting of control theory were first presented in [13]. They implicitly made critical use of the natural Zinbiel product structure of the space of iterated integral functionals. They have proved immensely useful for obtaining results for controllability and optimality, path-planning algorithms, and explicit normal forms for free nilpotent systems and nilpotent approximating systems. Similar explicit formula for the iterated integral functionals $\zeta_{\ell}$ were only found very recently [37], [38]. They are defined in terms of projections of the $\xi_{\ell}$ that make critical use of iterated convolution products of the projection map $I$ (which subtracts from every polynomial its constant part). The formulae in [37], [38] 
constitute generalizations of the Campbell-Baker-Hausdorff formula to "product of continuous families of exponentials. As opposed to [39], they are minimal in the sense of utilizing bases of the free Lie algebra, thereby guaranteeing a minimal number of terms.

\section{THE CHRONOLOGICAL POINT OF VIEW}

The formal group of exponential Lie series, in which the series of the controls take values naturally acts on $A(Z)$ by multiplication. In other words, every such series can be regarded as giving rise to a linear endomorphism of the algebras $A(Z)$ and $\hat{A}(Z)$ of all polynomials and of all series. As discussed in the preceding section, the group structure corresponds to multiplication in the algebra $A(Z)$, and in turn to concatenation of controls. Moreover, the algebra of endomorphisms of $A(Z)$ also is equipped with two convolution products, and naturally the question arises what the corresponding operations on controls are.

Here we are only considering very special endomorphisms, namely those given by multiplication by group-like elements. Other endomorphisms, that may be relevant to control, correspond to feedback operations, such as changes of bases of the distribution defined by the control vector field, and corresponding changes of basis in the space of control values.

Indeed, it turns out that the operation of adding controls in the sense the sum of two systems $\dot{x}=\sum_{a \in Z_{1}} u^{a} f_{a}$, and $\dot{x}=$ $\sum_{a \in Z_{2}} u^{a} f_{a}$ is given by $\dot{x}=\sum_{a \in Z_{1} \cup Z_{2}} u^{a} f_{a}$ corresponds to the convolution product $\star$.

This is a very common operation in control application. For example, an affine system with drift may be understood as the sum of an uncontrolled system $\left(Z_{1}=\{a\}, u^{a} \equiv\right.$ 1) and a driftless system. In other places one may have vector valued controls and needs to identify the interactions of the restricted systems each of which only utilize some components of the control.

This operation is intimately tied to the Zassenhaus formula which relates the exponential $\exp (X+Y)$ to a product of exponentials of $X, Y$, and their iterated Lie brackets. Full details of the correspondence of the sum of two systems and the convolution product will be reported in a different place. At this time, we add the observation that the other convolution product $\star^{\prime}$ is related to a dual perspective that views the action of group-like elements as multiplication operators on the space of linear functionals. In terms of controlled dynamical systems, these two perspectives would correspond to viewing the effect of controls on the points in the state space, and on the algebra of smooth functions.

\section{SERIES OF SYSTEMS AND THEIR PRODUCTS}

Again starting with the most abstract form of the ChenFliess series in the form $\sum_{w \in Z^{*}} w \otimes w$, one may as well focus on the other factor, partially evaluating this expression on the system data. One possible such choice is to consider a fixed set $\mathcal{F}=\left\{f_{a}: a \in Z\right\}$ of analytic vector fields on the state space $M$, and to evaluate the letters (generators of the algebra $A(Z)$ ) under the substitution homomorphism $\mathrm{Ev}_{\mathcal{F}}$ that sends a letter $a$ to a vector field $f_{a}: M \mapsto T M$ on the state space $M$.

$$
\left(\operatorname{Id} \otimes \operatorname{Ev}_{\mathcal{F}}\right)\left(\sum_{w \in Z^{*}} w \otimes w\right)=\sum_{w \in Z^{*}} w \otimes f_{w}
$$

This representation is of particular interest for systems that have finite, especially solvable and nilpotent Lie algebras.

The next series arise from further evaluating the above formal power series with coefficients that are high order partial differential operators on a given output functions of the system, and from even further evaluating these at a given initial point. The resulting noncommuting formal power series

$\left(\operatorname{Id} \otimes \operatorname{Ev}_{\mathcal{F}}\right)\left(\sum_{w \in Z^{*}} w \otimes w\right)(\varphi)\left(x_{0}\right)=\sum_{w \in Z^{*}} w \cdot\left(f_{w} \varphi\right)\left(x_{0}\right)$

now is a series with constant coefficients. It formally appears just like the series of a control, as used for the endpoint map - yet the algebra and control interpretation are different.

To make this series of a system directly useful for control interpretations, one maps the words on the left hand side to the iterated integrals (typically with variable upper limits) of input signals $u(t)$, and interprets the results as series expansions of the corresponding output signal $y(t)$.

Natural operations on systems are interconnections of various kinds, most notably parallel, cascade, and feedback connections. At the center of this work is the underlying composition product which has been studied in great detail by e.g. Gray and Li [24], [25] and Gray and Wang [11], [26]. These works address both the combinatorial and algebraic structures as well as the analytic (convergence) properties of such interconnections of input-output mappings that can be represented by Fliess operators. However, little appears to be known about the role of the Hopf algebra structure as described above and such series of systems. Just like the series of the controls discussed before, the series of systems can be multiplied and act by multiplication in analogous ways, including the two convolution products. Many open questions remain - but the main observation is that the two Hopf algebra structures with their two convolution products correspond to different points of view that consider the points in the state space, or the algebra of functions on it. Furthermore, the same underlying algebraic structures have different interpretations when looking at as systems and when looking at controls. 


\section{SUMMARY AND OUTLOOK}

This article lays out algebraic correspondences between abstract objects in combinatorial Hopf algebras and items of central importance in nonlinear control theory. The abstraction leads to deeper understanding of relationships, and has led to the discovery of simpler formulas that are of immediate utility in control. Recent discovery of explicit formulas for the $\zeta_{\ell}$ demonstrated the importance of underlying Hopf algebra structures in control. This article elaborates, in the context of control systems, the meaning of the convolution product which is so central to Hopf algebras. The article also points to many questions that remain open, many of whose answers may lead to deeper understanding of objects in control and to further simplifications of algorithms.

\section{ACKNOWLEDGMENTS}

The author would like the anonymous referees for their very careful reading of the original manuscript and for many valuable suggestions for improving it.

\section{REFERENCES}

[1] K.-T. Chen, "Integration of paths, geometric invariants and a generalized Baker-Hausdorff formula," Ann. of Math. (2), vol. 65, pp. 163178, 1957.

[2] M. Fliess, "Realizations of nonlinear systems and abstract transitive Lie algebras," Bull. Amer. Math. Soc. (N.S.), vol. 2, no. 3, pp. 444-446, 1980.

[3] M. Fliess, "Fonctionnelles causales non linéaires et indéterminées non commutatives," Bull. Soc. Math. France, vol. 109, no. 1, pp. 3-40, 1981.

[4] M. Fliess, "Réalisation locale des systèmes non linéaires, algèbres de Lie filtrées transitives et séries génératrices non commutatives," Invent. Math., vol. 71, no. 3, pp. 521-537, 1983.

[5] P. E. Crouch, "Solvable approximations to control systems," SIAM J. Control Optim., vol. 22, no. 1, pp. 40-54, 1984.

[6] B. Jakubczyk, "Existence and uniqueness of realizations of nonlinear systems," SIAM J. Control Optim., vol. 18, no. 4, pp. 455-471, 1980.

[7] B. Jakubczyk, "Local realizations of nonlinear causal operators," SIAM J. Control Optim., vol. 24, no. 2, pp. 230-242, 1986.

[8] B. Jakubczyk, "Realization theory for nonlinear systems: three approaches," in Algebraic and geometric methods in nonlinear control theory, vol. 29 of Math. Appl., pp. 3-31, Dordrecht: Reidel, 1986.

[9] H. J. Sussmann, "Lie brackets and local controllability: a sufficient condition for scalar-input systems," SIAM J. Control Optim., vol. 21, no. 5, pp. 686-713, 1983.

[10] Y. Wang and E. D. Sontag, "Generating series and nonlinear systems: analytic aspects, local realizability, and i/o representations," Forum Math., vol. 4, no. 3, pp. 299-322, 1992.

[11] W. S. Gray and Y. Wang, "Fliess operators on $L_{p}$ spaces: convergence and continuity," Systems Control Lett., vol. 46, no. 2, pp. 67-74, 2002.

[12] R. Ree, "Lie elements and an algebra associated with shuffles," Ann. of Math. (2), vol. 68, pp. 210-220, 1958.

[13] H. J. Sussmann, "A product expansion for the Chen series," in Theory and applications of nonlinear control systems (Stockholm, 1985), pp. 323-335, Amsterdam: North-Holland, 1986.

[14] J.-L. Loday, "Une version non commutative des algèbres de Lie: les algèbres de Leibniz," in R.C.P. 25, Vol. 44 (French) (Strasbourg, 1992), vol. 1993/41 of Prépubl. Inst. Rech. Math. Av., pp. 127-151, Strasbourg: Univ. Louis Pasteur, 1993.

[15] M. Kawski and H. J. Sussmann, "Noncommutative power series and formal Lie-algebraic techniques in nonlinear control theory," in Operators, systems, and linear algebra (Kaiserslautern, 1997), European Consort. Math. Indust., pp. 111-128, Stuttgart: Teubner, 1997.
[16] M.-P. Schützenberger, "Sur une propriété combinatoire des algèbres de lie libres pouvant être utilisée dans un probléme de mathématiques appliquées,", Séminaire P. Dubreil, Algèbres et Théorie des Nombres, 1958.

[17] K. Ebrahimi-Fard, D. Manchon, and F. Patras, "New identities in dendriform algebras," J. Algebra, vol. 320, no. 2, pp. 708-727, 2008

[18] K. Ebrahimi-Fard, D. Manchon, and F. Patras, "A magnus- and fertype formula in dendriform algebras," Found. Comput. Math.

[19] R. Grossman and R. G. Larson, "The realization of input-output maps using bialgebras," Forum Math., vol. 4, no. 2, pp. 109-121, 1992.

[20] R. Grossman and R. G. Larson, "Hopf-algebraic structure of combinatorial objects and differential operators," Israel J. Math., vol. 72, no. 1-2, pp. 109-117, 1990. Hopf algebras.

[21] R. Grossman and R. G. Larson, "Hopf-algebraic structure of families of trees," J. Algebra, vol. 126, no. 1, pp. 184-210, 1989.

[22] A. Murua, "The Hopf algebra of rooted trees, free Lie algebras, and Lie series," Found. Comput. Math., vol. 6, no. 4, pp. 387-426, 2006.

[23] C. Reutenauer, Free Lie algebras, vol. 7 of London Mathematical Society Monographs. New Series. New York: The Clarendon Press Oxford University Press, 1993. Oxford Science Publications.

[24] Y. Li and W. S. Gray, "The formal Laplace-Borel transform of Fliess operators and the composition product," Int. J. Math. Math. Sci., pp. Art. ID 34217, 14, 2006.

[25] W. S. Gray and Y. Li, "Generating series for interconnected analytic nonlinear systems," SIAM J. Control Optim., vol. 44, no. 2, pp. 646672 (electronic), 2005.

[26] W. S. Gray and Y. Wang, "Non-causal Fliess operators and their shuffle algebra," Internat. J. Control, vol. 81, no. 3, pp. 342-355, 2008.

[27] M. Kawski, "High-order small-time local controllability," in Nonlinear controllability and optimal control, vol. 133 of Monogr. Textbooks Pure Appl. Math., pp. 431-467, New York: Dekker, 1990.

[28] G. Stefani, "On the local controllability of a scalar-input control system," in Theory and applications of nonlinear control systems (Stockholm, 1985), pp. 167-179, Amsterdam: North-Holland, 1986.

[29] H. J. Sussmann, "A general theorem on local controllability," SIAM J. Control Optim., vol. 25, no. 1, pp. 158-194, 1987.

[30] W. Liu, "Averaging theorems for highly oscillatory differential equations and iterated Lie brackets," SIAM J. Control Optim., vol. 35, no. 6 , pp. 1989-2020, 1997.

[31] W. Liu, "An approximation algorithm for nonholonomic systems," SIAM J. Control Optim., vol. 35, no. 4, pp. 1328-1365, 1997.

[32] H. Z. Munthe-Kaas and W. M. Wright, "On the Hopf algebraic structure of Lie group integrators," Found. Comput. Math., vol. 8, no. 2, pp. 227-257, 2008.

[33] A. A. Agračev and R. V. Gamkrelidze, "Exponential representation of flows and a chronological enumeration,” Mat. Sb. (N.S.), vol. 107(149), no. 4, pp. 467-532, 639, 1978.

[34] A. A. Agračev and R. V. Gamkrelidze, "Chronological algebras and nonstationary vector fields," in Problems in geometry, Vol. 11 (Russian), pp. 135-176, 243, Vsesoyuz. Inst. Nauchn. i Tekhn. Informatsii, Moscow: Akad. Nauk SSSR, 1980.

[35] A. A. Agrachev and Y. L. Sachkov, Control theory from the geometric viewpoint, vol. 87 of Encyclopaedia of Mathematical Sciences. Berlin: Springer-Verlag, 2004. Control Theory and Optimization, II.

[36] M. Kawski, "The combinatorics of nonlinear controllability and noncommuting flows," in Mathematical control theory, Part 1, 2 (Trieste, 2001), ICTP Lect. Notes, VIII, pp. 223-311 (electronic), Abdus Salam Int. Cent. Theoret. Phys., Trieste, 2002.

[37] E. Gehrig, Hopf algebras, projections, and coordinates of the first kind in control theory. PhD thesis, Arizona State University, 2007.

[38] E. Gehrig and M. Kawski, "A Hopf-algebraic formula for compositions of noncommuting flows," in Proceedings IEEE Conference Decision and Control, (Cancun), pp. 156-1574, 2008.

[39] R. S. Strichartz, "The Campbell-Baker-Hausdorff-Dynkin formula and solutions of differential equations," J. Funct. Anal., vol. 72, no. 2, pp. 320-345, 1987. 